\title{
FRICTION EFFECT IN A PLANE PROBLEM OF PUNCH ACTING ON A HALF-SPACE WEAKENED BY CRACKS
}

\author{
Adam TOMCZYK \\ *Białystok University of Technology, Faculty of Mechanical Engineering, ul. Wiejska 45C, 15-351 Białystok, Poland \\ a.tomczyk@pb.edu.pl

\begin{abstract}
This work makes use of singular integral equations method to solve plane contact problem for a half-space containing cracks. This method is based on complex variables. Relationships are presented which help find the contact pressure under the punch and the value of stress intensity factors at the crack tips. A detailed solution is presented for the problem of a punch pressed to the surface of a half-space weakened by a single straight crack. It includes both a situation where the punch has a flat and parabolic base. The influence of friction and the position of the crack on the distribution of the values of stress intensity factors $\mathbf{K}_{\mathbf{I}}$ i $\mathbf{K}_{\mathbf{I I}}$ in the functions of crack length and distance from the contact zone was analyzed in detail.
\end{abstract}

Key words: Contact Problem, Elastic Half-Space, Crack, Stress Intensity Factor, Friction

\section{INTRODUCTION}

In the mechanical engineering or in the technology of abrasive machining, we often meet the problem connected with friction between cooperating elements. Many classic examples of such problems can be found in various friction brakes, gear units, in grinding processes, or in the problems concerning wheels rolling and braking on rails. In the analysis of such cases it is usually assumed that both elements have no defects, such as edge or internal cracks. It is obvious that the appearance of such defects leads to a redistribution and most of all strong concentration of stresses connected with the effect of a crack.

This work concentrates on the analysis of effects connected with the "sliding" of a punch pressed against an elastic half-space weakened by edge or internal cracks. The analysis makes use of the singular integral equations method including Muskhelishvili's complex potentials (Muskhelishvili, 1962). It covers a plane problem assuming that the punch is perfectly rigid and neglecting the problems of heat generation. In many papers, such problems are modeled by applying load in the form of an appropriate contact pressure on the surface of a half-space in the place where the punch is applied (Hills et al., 1993; Hills and Nowell, 1994). It can be also found for example in the series of works by Goshima and co-authors, which concentrate the problem of rolling (including sliding) of an rigid cylindrical punch on a damaged elastic halfspace. This analysis included both fatigue and temperature effects. The analyzed defects included a single edge crack extending to the surface of the elastic half-space (Goshima and Keer, 1990), two edge cracks of varying orientation (Goshima and Kamishima, 1996), a set of periodic cracks (Goshima and Kamishima, 1994; Goshima, 2003). We can also find the solution of a plane problem with a defect in the form of a single internal crack (Goshima and Soda, 1997) and problems with a threedimensional edge crack (Goshima, 2003; Goshima et al., 1990). Bryant et al. (1984) examined the interaction effects between cylindrical indenter and single-cracked half-space but only for the case when surface-braking crack was presented in contact zone. Other paper by Bryant (Keer and Bryant, 1983) presents a fatigue problem where cylindrical damaged indenter were considered or problem where a half-space is weakened by two cracks: vertical edge crack and horizontal subsurface crack (Keer et al., 1982). Similar subjects are covered in works by Hasebe et al., (Hasebe, 1981; Hasebe et al., 1989; Okumura et al., 1990; Hasebe and Qian, 1995, 1997, 1998; Qian and Hasebe, 1997), where for example a punch is applied with one rounded and one sharp end (Hasebe and Qian, 1998). The problem of contact between the punch and a half-space weakened by cracks was also analyzed by Panasyuk and co-authors (Panasyuk et al., 1995 2000; Datsyshyn et al., 2001), where trajectories are found for edge cracks in the context of fatigue (Datsyshyn et al., 2001). A detailed analysis has also been provided for the problem of a flat-based punch on the surface of a half-space weakened by a internal (subsurface) crack, where the faces of the crack are in contact (Panasyuk et al., 1995). For last years a problem of interaction between crack and punch has been applied to FGM materials. It was analyzed parabolic or cylindrical stamp acting on graded coatings (Guler and Erdogan, 2007) or the case when the base of the punch was assumed to be flat (Dag and Erdogan, 2002).

\section{FORMULATION OF THE PROBLEM}

Let us assume that into the $y=0$ surface of an elastic halfspace $-\infty<x<\infty, y \leq 0$ with internal cracks a punch of any shape (Fig. 1) is pressed with normal force $P$ and slide under the influence of tangential force $\rho P, \rho$ - friction coefficient. Let us also assume that under the influence of such loading the punch is in a limit equilibrium condition. Size of the contact zone between punch and surface of half-space is $2 a$. It should be noted that moment affects the punch preventing it from turning.

The boundary conditions on the $y=0$ plane can be noted as:

$$
\begin{aligned}
& \sigma_{x y}+\rho \sigma_{y}=0, \quad v=f(x)+C \\
& x \in L_{0}=\{x:-a<x<a\}
\end{aligned}
$$


$\sigma_{y}-i \sigma_{x y}=p_{0}(x)=0, \quad x \notin L_{0}$

where $\sigma_{y}, \sigma_{x y}$ are the normal and tangent component of the stress tensor, $v$ is a component of the displacement vector in the $y$ direction, $f(x)$ - a function describing the shape of the punch base, and $\mathrm{C}$ - constant (vertical displacement of the punch).

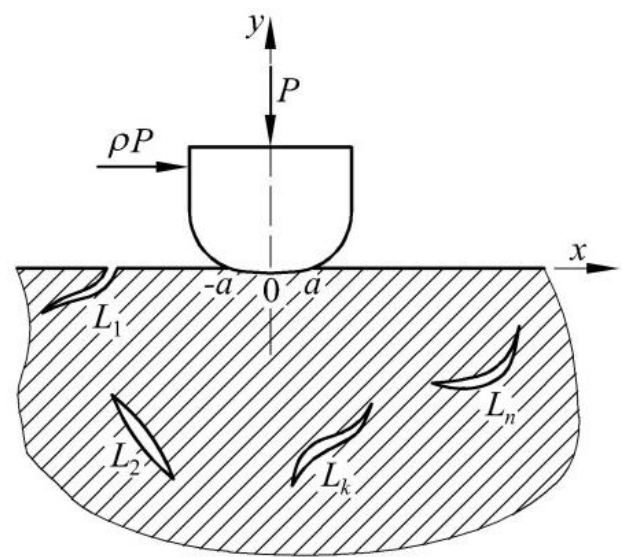

Fig. 1. Scheme of considered problem

The edgeses of the crack are assumed to be under a selfbalanced load in the following form:

$$
N^{ \pm}(t)+i T^{ \pm}(t)=p(t), \quad t \in L=\bigcup_{k=1}^{n} L_{k}
$$

where $N$ and $T$ are a normal and tangential component of the surface stress vector on the faces of the crack, $L_{k}(k=\overline{1, n})-$ the contour of $k$-th crack, "+" and "-" denote left and right edges of the $L_{k}$ contour respectively.

General complex stress potentials for a system of curved cracks in a half-space affected by external load, are as follows (Muskhelishvili, 1962; Savruk, 1981):

$$
\begin{aligned}
& \Phi_{1}(z)=\Phi_{0}(z)+\Phi(z), \quad \Psi_{1}(z)=\Psi_{0}(z)+\Psi(z) \\
& \Phi_{0}(z)=-\frac{1}{2 \pi i} \int_{L_{0}} \frac{p_{0}(t) \mathrm{d} t}{t-z} \\
& \Psi_{0}(z)=-\frac{1}{2 \pi i} \int_{L_{0}}\left[\frac{\overline{p_{0}(t)}}{t-z}-\frac{t p_{0}(t)}{(t-z)^{2}}\right] \mathrm{d} t \\
& \Phi(z)=\frac{1}{2 \pi} \int_{L}\left[\left(\frac{1}{t-z}-\frac{1}{\bar{t}-z}\right) g^{\prime}(t) \mathrm{d} t+\frac{t-\bar{t}}{(\bar{t}-z)^{2}} \overline{g^{\prime}(t)} \overline{\mathrm{d} t}\right] \\
& \Psi(z)=\frac{1}{2 \pi} \int_{L}\left[\left(\frac{1}{t-z}-\frac{1}{\bar{t}-z}-\frac{(t-\bar{t})(\bar{t}+z)}{(\bar{t}-z)^{3}} \overline{g^{\prime}(t)} \overline{\mathrm{d} t}-\right.\right. \\
& \left.-\left(\frac{\bar{t}}{(t-z)^{2}}-\frac{\bar{t}}{(\bar{t}-z)^{2}}\right) g^{\prime}(t) \mathrm{d} t\right]
\end{aligned}
$$

where $p_{0}$ denotes loading of the edge of the half-space and $g^{\prime}(t)$ is an unknown function on the contours of the cracks.

The derivative of vertical displacement of the edge of the halfspace can be given as follows (Gallin, 1980):

$$
\begin{aligned}
2 G \frac{\partial v(x, 0)}{\partial x}=- & \frac{\kappa-1}{2} \tau(x)+\frac{\kappa+1}{2 \pi} \int_{L_{0}} \frac{\sigma(t)}{t-z} \mathrm{~d} t+ \\
& +\operatorname{Im}\left[\kappa \Phi(x)-\overline{\Phi(x)}-x \overline{\Phi^{\prime}(x)}-\overline{\Psi(x)}\right]
\end{aligned}
$$

where $G$ is shear modulus, $v$-Poisson's ratio, $\kappa=3-4 v, \sigma$, $\tau-$ respectively: the normal and tangential component of the load on the surface of the half-space.

Satisfying the boundary conditions (1) with the Eq. (6) leads to the following integral equation:

$$
\begin{aligned}
& \varepsilon \rho \sigma(x)+\frac{1}{\pi} \int_{L_{0}} \frac{\sigma(t) \mathrm{d} t}{t-x}+2(\kappa+1)^{-1} \operatorname{Im}[\kappa \Phi(x)-\overline{\Phi(x)}- \\
& \left.-x \overline{\Phi^{\prime}(x)}-\overline{\Psi(x)}\right]=4 G(\kappa+1)^{-1} f^{\prime}(x), \quad x \in L_{0}
\end{aligned}
$$

where $\varepsilon=\frac{\kappa-1}{\kappa+1}$.

To Eq. (7) we have to add a punch equilibrium condition:

$\int_{L_{0}} \sigma(t) \mathrm{d} t=-P$

which guarantees uniqueness solution of the Eq. (7)

In the case of a homogeneous half-space, with no cracks, $\Phi(x)=\Psi(x)$, and Eqs. (7) and (8) are known (see Muskhelishvili, 1962).

Integral equations on the contours of the cracks can be written in condensed form (Savruk, 1981):

$\Phi_{1}\left(t^{\prime}\right)+\overline{\Phi_{1}\left(t^{\prime}\right)}+\frac{\overline{\mathrm{d} t^{\prime}}}{\mathrm{d} t}\left[t^{\prime} \overline{\Phi_{1}\left(t^{\prime}\right)}+\overline{\Psi_{1}\left(t^{\prime}\right)}\right]=p\left(t^{\prime}\right), \quad t^{\prime} \in L$,

where $\Phi_{1}\left(t^{\prime}\right)$ i $\Psi_{1}\left(t^{\prime}\right)$ - direct values of complex potentials (4).

To Eq. (9) we also have to add displacement uniqueness conditions while considering each internal crack:

$\int_{L_{k}} g^{\prime}(t) \mathrm{d} t=0, \quad k=\overline{2, n}$

Eqs. (7) and (9) form a system of singular integral equations of the first and second kind, which allows for the finding of normal contact pressure $\sigma(x)$ and a derivative of the displacement jumps vector $g^{\prime}(t)$ on the contours of cracks. Including conditions (8) and $(10)$ this system has a single solution for any right part in the function class, which have integrable singularities at the ends of the ranges of integration (Muskhelishvili, 1962).

It has to be noted that the system of integral Eqs. (7)-(9) was found under conditions (3). This means that there can be given loads on crack faces or, in a particular case, no load. Such boundary conditions can be realized only when the cracks are within the tensile stress fields. However, as a result of pressing the punch in, some or even all cracks can be found in the field of compression stresses, which is produced in the half-space under the punch. In that situation the faces of the cracks will be in contact. If this is so along the whole length of the crack, than Eqs (7)-(10) can be easily modifies, including the continuity of normal displacement on the contours of the crack and friction according to Amonton's law. In the general case we receive a mixed problem on the faces of the crack, which significantly increases the complexity of the solution. However, most works in this field, including this one, analyze these questions without including contact between crack faces. 
Complex potential (5) can be presented in the form:

$\Phi_{0}(z)=\frac{1}{\pi} \int_{L_{0}} f_{11}(t, z) \sigma(t) \mathrm{d} t$

$\Psi_{0}(z)=\frac{1}{\pi} \int_{L_{0}} h_{11}(t, z) \sigma(t) \mathrm{d} t$

$\Phi_{0}^{\prime}(z)=\frac{1}{\pi} \int_{L_{0}} g_{11}(t, z) \sigma(t) \mathrm{d} t$

$\Phi(z)=\frac{1}{\pi} \int_{L}\left[f_{21}(t, z) g^{\prime}(t) \mathrm{d} t+f_{22}(t, z) \overline{g^{\prime}(t)} \overline{\mathrm{d} t}\right]$

$\Psi(z)=\frac{1}{\pi} \int_{L}\left[h_{21}(t, z) g^{\prime}(t) \mathrm{d} t+h_{22}(t, z) \overline{g^{\prime}(t)} \overline{\mathrm{d} t}\right]$

$\Phi^{\prime}(z)=\frac{1}{\pi} \int_{L}\left[g_{21}(t, z) g^{\prime}(t) \mathrm{d} t+g_{22}(t, z) \overline{g^{\prime}(t)} \overline{\mathrm{d} t}\right]$

where:

$f_{11}(t, z)=-\frac{1+i \rho}{2 i} \frac{1}{t-z}, \quad f_{21}(t, z)=\frac{1}{2}\left(\frac{1}{t-z}-\frac{1}{\bar{t}-z}\right)$

$f_{22}(t, z)=\frac{t-\bar{t}}{2(\bar{t}-z)^{2}}, \quad g_{11}(t, z)=-\frac{1+i \rho}{2 i} \frac{1}{(t-z)^{2}}$

$g_{21}(t, z)=\frac{1}{2}\left(\frac{1}{(t-z)^{2}}-\frac{1}{(\bar{t}-z)^{2}}\right), \quad g_{22}(t, z)=\frac{t-\bar{t}}{(\bar{t}-z)^{3}}$

$h_{11}(t, z)=-\frac{1}{2 i}\left[\frac{1-i \rho}{t-z}-\frac{t(1+i)}{(t-z)^{2}}\right]$

$h_{21}(t, z)=-\frac{\bar{t}}{2}\left(\frac{1}{(t-z)^{2}}-\frac{1}{(\bar{t}-z)^{2}}\right)$

$h_{22}(t, z)=\frac{1}{2}\left(\frac{1}{t-z}-\frac{1}{\bar{t}-z}-\frac{(t-\bar{t})(\bar{t}+z)}{(\bar{t}-z)^{3}}\right)$

Taking into account Eqs. (11) and (12), the system of integral Eqs. (7) and (9) can be noted as:

$$
\begin{gathered}
\varepsilon \rho \sigma(x)+\frac{1}{\pi} \int_{L_{0}} \frac{\sigma(t) \mathrm{d} t}{t-x}+\frac{1}{\pi} \operatorname{Im} \int_{L}\left[K_{1}(t, x) g^{\prime}(t) \mathrm{d} t+\right. \\
\left.+L_{1}(t, x) \overline{g^{\prime}(t)} \overline{\mathrm{d} t}\right]=\frac{4 G}{\kappa+1} f^{\prime}(x), \quad x \in L_{0} \\
\frac{1}{\pi} \int_{L}\left[K_{2}\left(t, t^{\prime}\right) g^{\prime}(t) \mathrm{d} t+L_{2}\left(t, t^{\prime}\right) \overline{g^{\prime}(t)} \overline{\mathrm{d} t}\right]+ \\
+\frac{1}{\pi} \int_{L_{0}} M\left(t, t^{\prime}\right) \sigma(t) \mathrm{d} t=p\left(t^{\prime}\right), \quad t^{\prime} \in L
\end{gathered}
$$

Kernels of the system of Eqs. (13) and (14) are given by functions $f_{i j}, g_{i j}$ and $h_{i j}(i, j=1,2)$ described by relations (12), can be represent as follows:

$$
\begin{aligned}
& K_{1}(t, x)=\frac{2}{\kappa+1}\left[\kappa f_{21}(t, x)-\overline{f_{22}(t, x)}-x \overline{g_{22}(t, x)}-\overline{h_{22}(t, x)}\right] \\
& L_{1}(t, x)=\frac{2}{\kappa+1}\left[\kappa f_{22}(t, x)-\overline{f_{21}(t, x)}-x \overline{g_{21}(t, x)}-\overline{h_{21}(t, x)}\right] \\
& K_{2}\left(t, t^{\prime}\right)=f_{21}\left(t, t^{\prime}\right)+\overline{f_{22}\left(t, t^{\prime}\right)}+\frac{\overline{\mathrm{d} t^{\prime}}}{\mathrm{d} t}\left[t^{\prime} \overline{g_{22}\left(t, t^{\prime}\right)}+\overline{h_{22}\left(t, t^{\prime}\right)}\right] \\
& L_{2}\left(t, t^{\prime}\right)=f_{22}\left(t, t^{\prime}\right)+\overline{f_{21}\left(t, t^{\prime}\right)}+\frac{\overline{\mathrm{d} t^{\prime}}}{\mathrm{d} t}\left[t^{\prime} \overline{g_{21}\left(t, t^{\prime}\right)}+\overline{h_{21}\left(t, t^{\prime}\right)}\right] \\
& M\left(t, t^{\prime}\right)=f_{11}\left(t, t^{\prime}\right)+\overline{f_{11}\left(t, t^{\prime}\right)}+\frac{\overline{\mathrm{d} t^{\prime}}}{\mathrm{d} t}\left[t^{\prime} \overline{g_{11}\left(t, t^{\prime}\right)}+\overline{h_{11}\left(t, t^{\prime}\right)}\right]
\end{aligned}
$$

Note, that function $\mathrm{K}_{2}\left(\mathrm{t}, \mathrm{t}^{\prime}\right)$ includes Cauchy's singular kernel. This part will not be specially singled out, unless numerical integration formulas will be used to carry out the numerical calculations, which are correct for both singular and regular integrals.

\section{PARTICULAR CASE OF A SINGLE EDGE CRACK}

Let us consider a case, where a half-space is weakened by only one straight crack (Fig. 2) Let us introduce a parametric notation of the contour of crack $L$ and the zone of contact between the punch and the surface of half-space $L_{0}$ :

$t=a \xi, \quad x=a \eta, \quad-1<\xi, \eta<1, \quad\left(t, x \in L_{0}\right)$

$t=a \omega(\xi), \quad t^{\prime}=a \omega(\eta), \quad-1<\xi, \eta<1, \quad\left(t, t^{\prime} \in L\right)$

$\omega(\xi)=-b^{*}-\frac{l^{*}}{2}(\xi+1) e^{i \varphi}, \quad b^{*}=\frac{b}{a}, \quad l^{*}=\frac{l}{a}$ where $\varphi$ is angle of crack orientation defined according to Fig. 2.

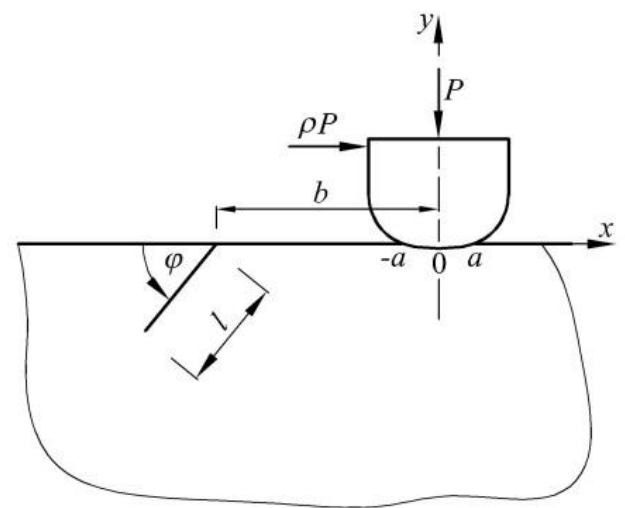

Fig. 2. Scheme of a pressed punch „sliding” on a surface of a single-cracked half-space

Moving to non-dimensional variables and functions the system of integral Eqs. (13) and (14) and the condition of solvability (8) has the following form:

$$
\begin{aligned}
& \varepsilon \rho \sigma(\eta)+\frac{1}{\pi} \int_{-1}^{1} \frac{\sigma(\xi) \mathrm{d} \xi}{\xi-\eta}+\frac{1}{\pi} \operatorname{Im} \int_{-1}^{1}\left[K_{1}(\xi, \eta) g^{\prime}(\xi)+\right. \\
& \left.+L_{1}(\xi, \eta) \overline{g^{\prime}(\xi)}\right] \mathrm{d} \xi=F(\eta), \quad|\eta|<1
\end{aligned}
$$




$$
\begin{aligned}
& \frac{1}{\pi} \int_{-1}^{1}\left[K_{2}(\xi, \eta) g^{\prime}(\xi)+L_{2}(\xi, \eta) \overline{g^{\prime}(\xi)}\right] \mathrm{d} \xi+ \\
& \quad+\frac{1}{\pi} \int_{-1}^{1} M(\xi, \eta) \sigma(\xi) \mathrm{d} \xi=p(\eta), \quad|\eta|<1 \\
& \int_{-1}^{1} \sigma(\xi) \mathrm{d} \xi=A=\frac{-P}{a},
\end{aligned}
$$

where:

$$
\begin{aligned}
& \sigma(a \eta)=\sigma(\eta), \quad g^{\prime}(a \omega(\xi)) \omega^{\prime}(\xi)=g^{\prime}(\xi) \\
& p(a \omega(\xi))=p(\eta), \quad 4 G(\kappa+1)^{-1} f^{\prime}(a \eta)=F(\eta) \\
& a K_{1}(a \omega(\xi), a \eta)=K_{1}(\xi, \eta) \\
& a L_{1}(a \omega(\xi), a \eta)=L_{1}(\xi, \eta) \\
& a K_{2}(a \omega(\xi), a \omega(\eta))=K_{2}(\xi, \eta) \\
& a L_{2}(a \omega(\xi), a \omega(\eta))=L_{2}(\xi, \eta) \\
& a M(a \xi, a \omega(\eta))=M(\xi, \eta)
\end{aligned}
$$

A solution for the system of integral Eqs. (17)-(18), which satisfies condition (19) will be sought in the class of functions, which have integrable singularities:

$$
\begin{aligned}
& \sigma(\xi)=w(\xi) q(\xi) \\
& w(\xi)=(1-\xi)^{\alpha}(1+\xi)^{\beta}, \quad-1<\alpha, \beta<0 \\
& g^{\prime}(\xi)=\left(1-\xi^{2}\right)^{-1 / 2} u(\xi)
\end{aligned}
$$

where $q(\xi)$ and $u(\xi)$ are continuous functions in a closed interval $[-1,1]$, and parameters $\alpha$ and $\beta$ are roots $\alpha=-0.5+\mu, \beta=-0.5-\mu, \mu=\arctan \varepsilon \rho$ of characteristic equations: $\cot \pi \alpha=-\varepsilon \rho$, $\cot \pi \beta=\varepsilon \rho$.

Let us remind that only for internal cracks condition (10) must be satisfied. However for the case of the edge crack singularity is produced only at the one crack tip (i.e. for $\xi=+1$ ). At the second tip $(\xi=-1)$, which extends to the surface, singularity disappears and also condition (10) can not be satisfied. So the solution of Eqs. (17)-(18) will be sought in the same class of functions (21) but condition (10) should be replaced by:

$u(-1)=0$

In the case of a homogeneous half-space, with no cracks, there is $g^{\prime}(\hat{\xi})=0$ and a system of integral Eqs. (17) and (18) is reduced to a single Eq. (18) when condition (19) is satisfy.

\section{NUMERICAL SOLUTION OF SINGULAR INTEGRAL EQUATIONS SYSTEM}

For a numerical solution of the system of Eqs. (17)-(19) we will use the quadrature method (or the mechanical quadrature method) (Savruk, 1981; Savruk et al., 1999, Savruk and Tomczyk, 2010) using Gauss-Chebyshev nodes. As a result we receive the following system of linear algebraic equations:

$$
\begin{array}{r}
\sum_{k=1}^{n} a_{m k} q\left(\xi_{k}\right)+\operatorname{Im} \sum_{k=1}^{\hat{n}}\left[b_{1 m k} u\left(\widehat{\xi}_{k}\right)+c_{1 m k} \overline{u\left(\hat{\xi}_{k}\right)}\right]=F\left(\eta_{m}\right), \\
m=1, \ldots, n-1 \\
\sum_{k=1}^{\hat{n}}\left[b_{2 m k} u\left(\widehat{\xi}_{k}\right)+c_{2 m k} \overline{u\left(\widehat{\xi}_{k}\right)}\right]+\sum_{k=1}^{n} d_{m k} q\left(\xi_{k}\right)=p\left(\widehat{\eta}_{m}\right), \\
m=1, \ldots, \hat{n}-1
\end{array}
$$

$\sum_{k=1}^{n} a_{n k} q\left(t_{k}\right)=A, \quad \sum_{k=1}^{\bar{n}}(-1)^{k} \sqrt{\frac{1-\xi_{k}}{1+\xi_{k}}} u\left(\xi_{k}\right)=0$

$\xi_{k}=\cos \frac{2 k-1}{2 n} \pi, \quad \bar{\xi}_{k}=\cos \frac{2 k-1}{2 \widehat{n}} \pi$

$\eta_{m}=\cos \frac{\pi m}{n}, \quad \hat{\eta}_{m}=\cos \frac{\pi m}{\hat{n}}$

The unknown values here are functions $q(\xi)$ and $u(\xi)$ respectively in nodes $\xi_{k}$ and $\hat{\xi}_{k}$. The coefficients of the unknowns in the system of Eqs. (23) are given by the following formulas:

$$
\begin{aligned}
& a_{m k}=-\frac{1}{n}(-1)^{k} \sqrt{1-\xi_{k}^{2}}\left\{\frac{X_{0}}{1-\eta_{m}}\left[\frac{1}{1-\xi_{k}}-\frac{T_{n}\left(\eta_{m}\right)}{\eta_{m}-\xi_{k}}\right]+\right. \\
& \left.+\frac{R_{0}}{1+\eta_{m}}\left[\frac{(-1)^{n}}{1+\xi_{k}}+\frac{T_{n}\left(\eta_{m}\right)}{\eta_{m}-\xi_{k}}\right]+X_{3}\left(\eta_{m}\right)+X_{4}\left(\eta_{m}\right) \frac{T_{n}\left(\eta_{m}\right)}{\eta_{m}-\xi_{k}}\right\} \\
& a_{n k}=\frac{\pi}{n} \sqrt{1-\xi_{k}^{2}}\left[w\left(\xi_{k}\right)-(-1)^{k} \frac{X_{0}}{1-\xi_{k}}+(-1)^{k+n} \frac{R_{0}}{1+\xi_{k}}\right] \\
& b_{1 m k}=\frac{1}{\hat{n}} K_{1}\left(\bar{\xi}_{k}, \eta_{m}\right), \quad b_{2 m k}=\frac{1}{\widehat{n}} K_{2}\left(\bar{\xi}_{k}, \hat{\eta}_{m}\right) \\
& c_{1 m k}=\frac{1}{\hat{n}} L_{1}\left(\bar{\xi}_{k}, \eta_{m}\right), \quad c_{2 m k}=\frac{1}{\hat{n}} L_{2}\left(\bar{\xi}_{k}, \hat{\eta}_{m}\right) \\
& d_{m k}=\frac{1}{n} \sqrt{1-\xi_{k}^{2}}\left[M\left(\xi_{k}, \widehat{\eta}_{m}\right) w\left(\xi_{k}\right)-\right. \\
& \left.-(-1)^{k} \frac{X_{0} M\left(1, \hat{\eta}_{m}\right)}{1-\xi_{k}}+(-1)^{n+k} \frac{R_{0} M\left(-1, \hat{\eta}_{m}\right)}{1+\xi_{k}}\right] \\
& X_{0}=\frac{1}{2}\left[\frac{2 \alpha}{\sin \pi \alpha}-\frac{1}{n} \sum_{k=1}^{n} X\left(\xi_{k}\right) \sqrt{1-\xi_{k}^{2}}\right] \\
& R_{0}=\frac{1}{2}\left[\frac{2 \beta}{\sin \pi \beta}-\frac{1}{n} \sum_{k=1}^{n} R\left(\xi_{k}\right) \sqrt{1-\xi_{k}^{2}}\right] \\
& X_{1}(x)=\frac{1}{2}\left[X(x) \cot \pi \alpha-\frac{1}{\sin \pi \alpha}-R(x) \cot \pi \beta+\frac{1}{\sin \pi \beta}\right] \\
& X_{3}(x)=\frac{1}{n} \sum_{r=1}^{n} w\left(\xi_{r}\right) \sqrt{1-\xi_{r}^{2}} F\left(x, \xi_{r}, \xi_{k}\right) \\
& X_{4}(x)=\varepsilon \rho w(x)+X_{1}(x) \\
& X(x)=\left(\frac{1-x}{1+x}\right)^{\alpha}, R(x)=\left(\frac{1+x}{1-x}\right)^{\beta} \\
& F\left(x, \xi_{r}, \xi_{k}\right)=\left\{\begin{array}{l}
\frac{T_{n}(x)}{\left(x-\xi_{k}\right)\left(x-\xi_{r}\right)}, \quad x \neq \xi_{k}, x \neq \xi_{r}, \xi_{k} \neq \xi_{r} \\
-\frac{n(-1)^{r}}{\sqrt{1-\xi_{r}^{2}}\left(\xi_{r}-x\right)}+\frac{T_{n}(x)}{\left(\xi_{r}-x\right)^{2}}, x \neq \xi_{k}=\xi_{r}
\end{array}\right.
\end{aligned}
$$

(1)


where $\operatorname{Tn}(x)=\cos (n \arccos x)$ is a first kind Chebyshev polynomial of $n$ degree.

Using Lagrange's interpolating polynomial for nodes $\xi_{k}$ we can find the values of function $q(\xi)$ at any point:

$q(\xi)=-\frac{1}{n} \sum_{k=1}^{n}(-1)^{k} q\left(\xi_{k}\right) \sqrt{1-\xi_{k}^{2}} \frac{T_{n}(x)}{\xi-\xi_{k}}$

Particularly, at the ends of the interval $[-1,1]$ we receive:

$q( \pm 1)=\mp \frac{( \pm 1)^{n}}{n} \sum_{k=1}^{n}(-1)^{k} \sqrt{\frac{1 \pm \xi_{k}}{1 \mp \xi_{k}}} q\left(\xi_{k}\right)$

Similarly, we search for the values of function $u\left(\hat{\xi}_{\mathrm{k}}\right)$ in nodes $\widehat{\xi}_{\mathrm{k}}$ :

$u(\bar{\xi})=-\frac{1}{n} \sum_{k=1}^{n}(-1)^{k} u\left(\xi_{k}\right) \sqrt{1-\bar{\xi}_{k}^{2}} \frac{T_{n}(x)}{\bar{\xi}-\bar{\xi}_{k}}, \quad-1 \leq \bar{\xi} \leq 1$

$u( \pm 1)=\mp \frac{( \pm 1)^{n}}{n} \sum_{k=1}^{n}(-1)^{k} \sqrt{\frac{1 \pm \bar{\xi}_{k}}{1 \mp \bar{\xi}_{k}}} u\left(\xi_{k}\right)$

Stress intensity factors at the crack tip $\hat{\xi}=1\left(K_{\mathrm{I}, \mathrm{II}}^{+}\right)$are found with these formulas (Savruk, 1981):

$K_{\mathrm{I}}^{+}-i K_{\mathrm{II}}^{+}=-\sqrt{\pi a\left|\omega^{\prime}(1)\right|} \frac{u(1)}{\omega^{\prime}(1)}$

It has to be noted that in the case of a flat-based punch contact zone $L_{0}$ is known. However, if the punch base is rounded at one or both ends, the dimensions of the contact zone are not known. In these cases the stresses at both ends of the contact zone are finite and for the purpose of finding the location of this zone we have one or two additional conditions. Particularly, for the parabolic punch, which also was the subject of our research, we have additional conditions:

$q(-1)=q(1)=0$

thus we determine the half width of contact zone $a$ and the eccentricity $e$ (distance of the center of these zone from the punch axis).

\section{ANALYSIS OF RESULTS}

The theoretical relations presented in the previous paragraph were used to create numerical algorithms which allow to determine the distribution of contact pressure and the values of stress intensity factors at the crack tip. As it was already mentioned, two different shapes of the base of the punch were considered - flat and parabolic. It was analyzed in detail the influence of the shape of the punch base on the distribution of dimensionless stress intensity factors $F_{\mathrm{I}}=\frac{K_{\mathrm{I}} \sqrt{a}}{P}, F_{\mathrm{II}}=\frac{K_{\mathrm{II}} \sqrt{a}}{P}$. It was assumed in the numerical calculations that the edges of the crack are free of load $(N=T=0$ in Eq. (3)).

Results of numerical analysis prove that existence of crack (or set of cracks) has a significant influence on contact pressure distribution (see Fujimoto et al, 1992, Tomczyk 2011; Savruk and Tomczyk, 2010). It corresponds with both flat and parabolic punch particularly for crack orientation angles from the range $\left(90^{\circ}, 180^{\circ}\right)$. For these angles crack tip can be found in material under contact zone directly and produce a "rapidly" perturbation of typical contact pressure distribution. Assuming regular contact pressure distribution in the place where punch acting can produce considerable errors in many contact problems. Only taking into consideration a mutual interaction between crack (or set of cracks) and punch gives a detailed information about character of pressure distribution. This interaction can be neglected for the case of homogenous half-space or when the crack is far away from the punch. As it was mentioned for the case of flat punch contact zone size $a$ is known. For parabolic punch this size should be obtained by using condition of pressure reducing to zero in the ends of contact zone (see Eq. (32)).

In present analysis the dependence of stress intensity factors values on distance between crack and punch, crack length and its orientation and friction coefficient is discussed. It is also analysed the effect of punch shape (parabolic or flat) on stress intensity factors.

In the case of no friction or for small values of friction coefficient crack located close to the punch can be present in fields of compression stresses. Situation like this can take place for both: when $0^{\circ}<\varphi<90^{\circ}$ (Fig. 3a), and when $90^{\circ}<\varphi<180^{\circ}$ (Fig. 3b).

a)
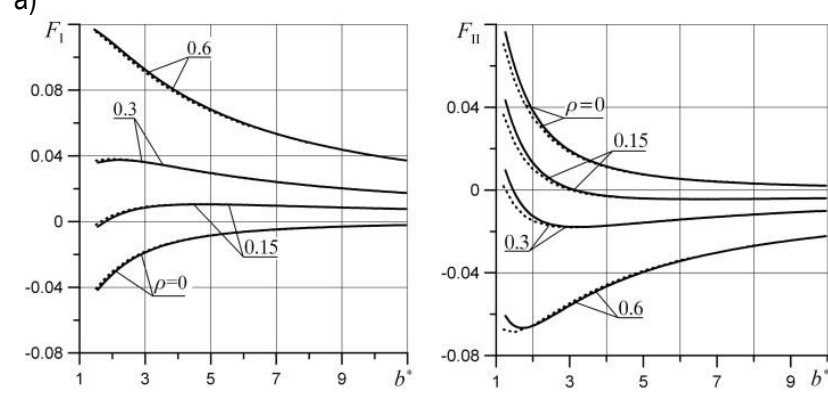

b)
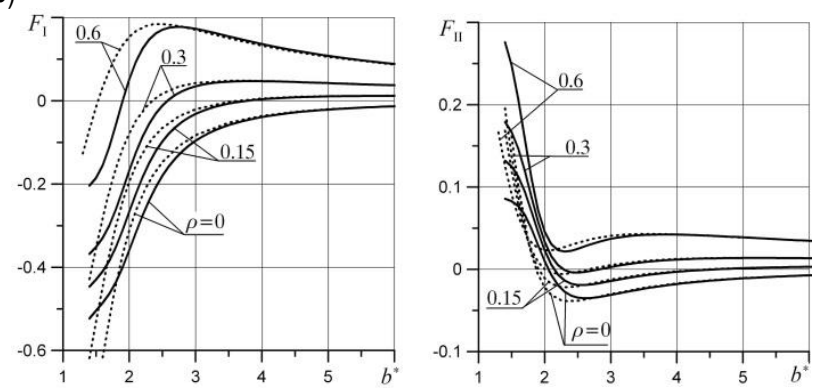

Fig 3. The effect of distance between crack and punch on dimensionless stress intensity factors $F_{I}$ and $F_{I I}$ for different values of friction coefficient $\left(l^{*}=1, v=0.3\right)$ : a) $\varphi=45^{\circ}$, b) $\varphi=135^{\circ}$

(dashed lines - parabolic punch, continuous lines - flat punch)

The effect of punch shape is distinctly seen for $\varphi \in\left(90^{\circ}, 180^{\circ}\right)$ particular for close cracks when $b^{*}<2.5$ (Fig. 3b, Fig. 4). For cracks defined by $0^{\circ}<\varphi<90^{\circ}$ the effect of punch shape can be neglected even for small values of distance between crack and punch. When the suitable conditions exist the crack can close when the flat punch is acting while for the case of parabolic punch the same crack is opened. The effect like this is more probable when the angle $\varphi$ is close to $180^{\circ}$ and values of $b^{*}$ are very small (Fig. 5).

A very interesting situation we can observe in the Fig. 6 . It presents the effect of translation of crack tip into material under 
contact zone directly produced by increasing in crack length. It is clearly seen that crack starts to close for certain values of $l^{*}$. This effect appears for small values of friction coefficient earlier than for higher values. Increasing in friction delays crack closure process. Let us note that there is no any influence of punch shape on stress intensity factors in Fig. 6.

a)
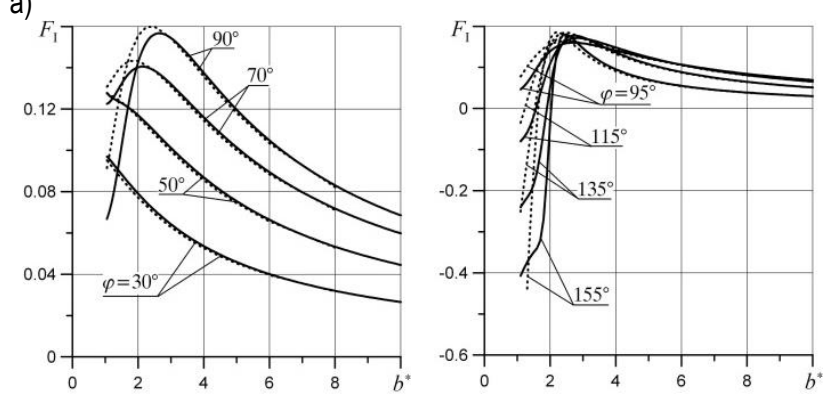

b)
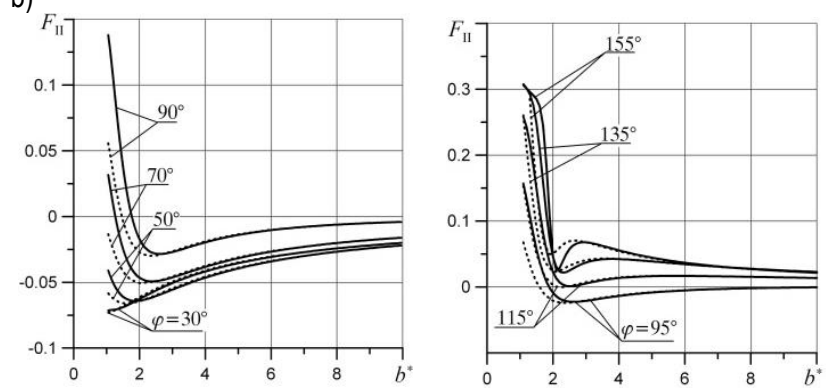

Fig. 4. The effect of distance between crack and punch on dimensionless stress intensity factors $F_{I}(a)$ and $F_{\| I}(b)$ for various crack orientations $(l=1, v=0.3, \rho=0.6)$

(dashed lines - parabolic punch, continuous lines - flat punch)

a)
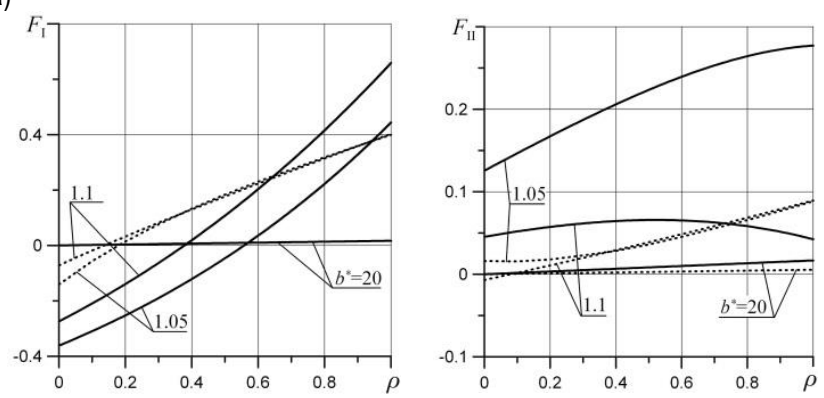

b)
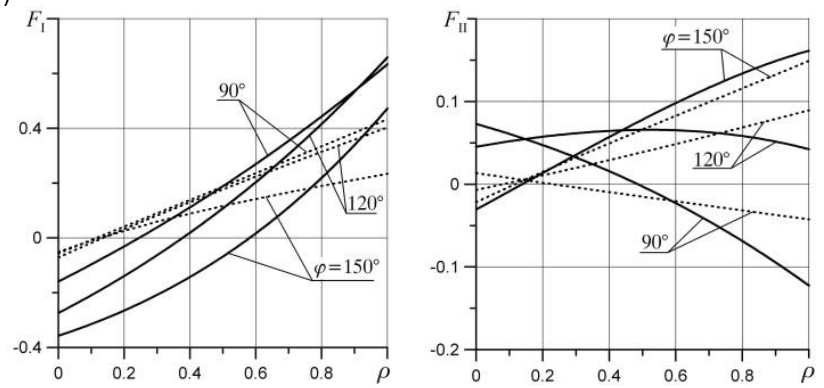

Fig. 5. Influence of friction on dimensionless stress intensity factors $F$ and $F_{\|}$for flat (continuous lines) and parabolic (dashed lines) punch: a) the effect of distance between crack and punch $\left(I^{*}=0.1, v=0.3, \varphi=120^{\circ}\right)$,

b) the effect of crack orientation $\left(I^{*}=0.1, b^{*}=1.1, v=0.3\right)$ a)

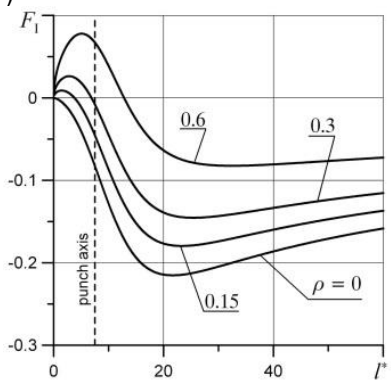

b)

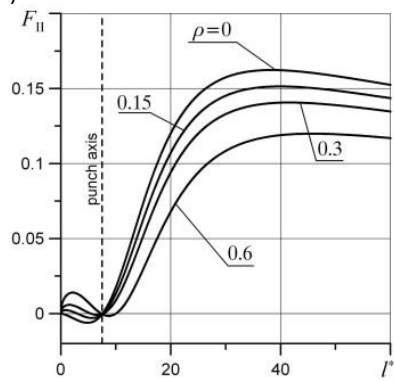

Fig. 6. The effect of crack length on dimensionless stress intensity factors $F_{I}(a)$ i $F_{I I}(b)$ for various values of friction coefficient $\left(b^{*}=15, v=0.3, \varphi=120^{\circ}\right)$

a)

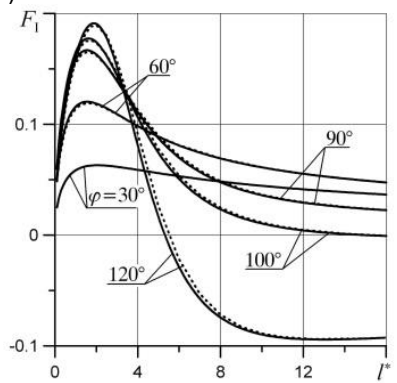

b)

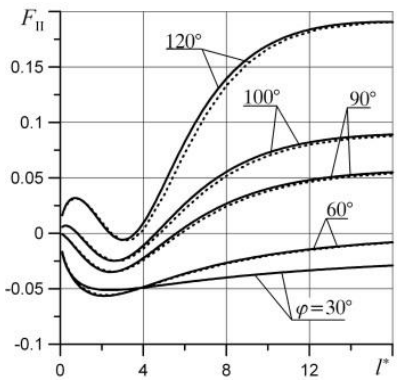

Fig. 7. The effect of crack length on dimensionless stress intensity factors $F_{\mathrm{I}}(\mathrm{a})$ i $F_{\mathrm{II}}(\mathrm{b})$ for various crack orientations $\left(b^{*}=5, v=0.3, \rho=75\right)$ (dashed lines - parabolic punch, continuous lines - flat punch)

a)
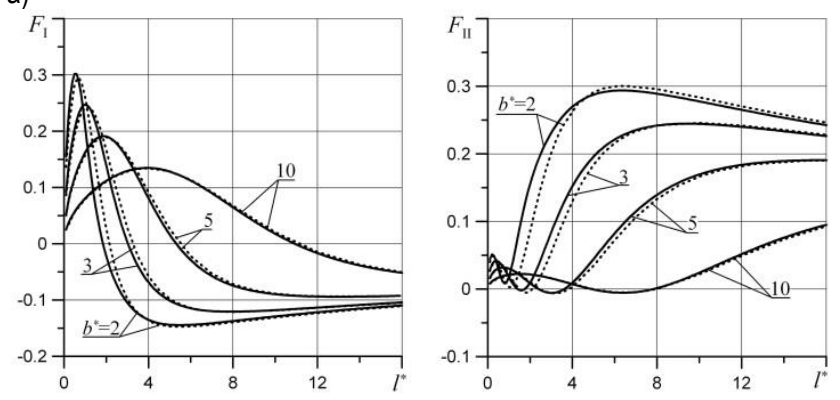

b)
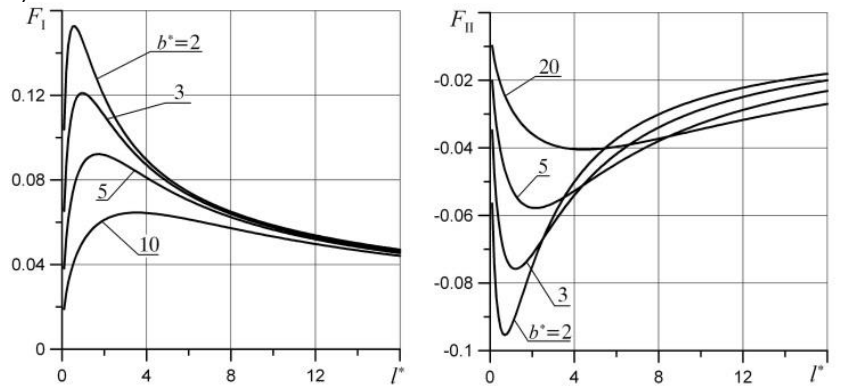

Fig. 8. The effect of crack length on dimensionless stress intensity factors $F_{1}$ and $F_{11}$ for various values of distance between crack and punch $(\rho=0.75, v=0.3)$ : a) $\varphi=120^{\circ}$, b) $\varphi=45^{\circ}$

(dashed lines - parabolic punch, continuous lines - flat punch)

It is very characteristic for $90^{\circ}<\varphi<180^{\circ}$ that factors $F_{1}$ and $F_{\|}$decrease to zero considerably slower (Fig. 8a) than for $0^{\circ}<\varphi<90^{\circ}$ (Fig. 8b). The effect of crack closure can be achieved much more earlier for higher values of $\varphi$ (Fig. 7a). This 
effect appears more distinctly for $90^{\circ}<\varphi<180^{\circ}$. For the case of $0^{\circ}<\varphi<90^{\circ}$ it can be neglected particularly for small values of $\varphi$ when it is not visible.

The effect of crack length is very strong in the region of short cracks where $l^{*}<6$ (Fig. 8). The most dangerous situations appear not for shortest cracks but for cracks where stress intensity factors reaches maximal values. This characteristic length depends on distance between crack and punch mainly and increases as $b^{*}$ increases. The effect of punch shape is as clearly as crack is located closer to the punch and for $90^{\circ}<\varphi<180^{\circ}$ only.

\section{CONCLUSIONS}

This work presents a solution of the problem of a rigid punch acting on a half-space weakened by a set of cracks. In the analysis the method of singular integral equations has been used. It has been analyzed in detail the case of a half-space weakened by a single edge crack while the base of the punch is parabolic or flat. Therefore it has been observed the influence of shape of the base of the punch on the the values of $K_{I}$ and $K_{\| I}$. This influence can be neglected for the crack orientation angle $\varphi \in\left(0^{\circ}, 90^{\circ}\right)$ as opposed to $\varphi \in\left(90^{\circ}, 180^{\circ}\right)$. For a numerical solution of the system of integral equations the quadrature method has been used, which allowed to reduce this system to a system of linear algebraic equations.

Presented method gives possibility for simultaneously calculating the contact pressure and the values of stress intensity factors. This means that it is possible to take into account the mutual interaction between crack and punch. While analyzing the influence of the punch shape, it has to be noted that it has a significant influence not only on the characteristics of contact pressure distribution, but also on the values of stress intensity factors. This influence is particularly notable for cracks close to the contact zone and decreases as the crack "moves away". So it shows that for far cracks it doesn't matter a character of applied loading: parabolic, asymptotic or even in the form of concentrate force. On the other hand for close cracks it should be noted that even not accounting for friction the shape of the punch is not without importance. Furthermore, it was made a detailed analysis of the influence of the orientation and length of the crack on the values of stress intensity factors $K_{\iota}$ and $K_{\| l}$. For vertically oriented cracks the shape of the punch has no influence on the values of stress intensity factors even for close cracks. Proposed method also allows for description of the changes in the contact zone size and the eccentricity of its center when the parabolic punch is applied.

The method presented here can be used for the analysis of problems concerning a half-space weakened by a set of edge or internal cracks. An attempt could also be made to use this solution to analyze problems of single cracks or sets of cracks under the surface of a half-space or internal curvelinear macroinclusions or holes. Furthermore, the singular integral equations method may be used in future to solve problems of bilateral contact, with the assumption that both the half-space and the punch are not rigid.

\section{REFERENCES}

1. Bryant M.D., Miller G.R., Keer L.M. (1984), Line Contact Between a Rigid Indenter and a Damaged Elastic Body, Q. J. Mechanics Appl. Mathematics, Vol. 37, 467-478.

2. Dag S., Erdogan F. (2002), A Surface Crack in a Graded Medium Loaded by a Sliding Rigid Stamp, Engng Fract. Mech., Vol. 69, 17291751.

3. Datsyshyn O.P., Panasyuk V.V., Pryshlak R.E., Terlets'kyi A.B. (2001), Paths of edge cracks in rolling bodies under the Conditions of Boundary Lubrication, Materials Science, Vol. 37: 363-373.

4. Fujimoto K., Ito H., Yamamoto T. (1992), Effect of Cracks on the Contact Pressure Distribution, Tribology Transactions, Vol. 37, 683695.

5. Galin L.A. (1980), Contact Problems of the Theory of Elasticity and Viscoelasticity, Nauka, Moscow, (in Russian).

6. Goshima T. (2003), Thermomechanical Effects on Crack Propagation in Rolling Contact Fatigue Failure, Journal of Thermal Stresses, Vol. 26, 615-639.

7. Goshima T., Hanson M.T., Keer L.M. (1990), Three-Dimensional Analysis of Thermal Effects on Surface Crack Propagation in Rolling Contact, Journal of Thermal Stresses, Vol. 13, 237-261.

8. Goshima T., Kamishima Y. (1994), Mutual Interference of Multiple Surface Cracks Due to Rolling-Sliding Contact with Frictional Heating, JSME International Journal Series A, Vol. 37: 216-223.

9. Goshima T., Kamishima Y. (1996), Mutual interference of Two Surface Cracks in Semi-Infinite Body Due to rolling contact with Frictional Heating by a Rigid Roller, JSME International Journal Series A, Vol. 39, 26-33.

10. Goshima T., Keer L.M. (1990), Thermoelastic Contact Between a Rolling Rigid Indenter and a Damaged Elastic Body, Journal of Tribology, Transactions of the ASME, Vol. 112, 382-391.

11. Goshima T., Soda T. (1997), Stress Intensity Factors of a Subsurface Crack in a Semi-Infinite Body Due to Rolling/Sliding Contact and Heat Generation, JSME International Journal Series A 40, 263-270.

12. Guler M.A., Erdogan F. (2007), Frictional Sliding Contact Problems of Rigid Parabolic and Cylindrical Stamps on Graded Coatings, International Journal of Mechanical Sciences, Vol. 49, 161-182,

13. Hasebe N. (1981), An Edge Crack in a Semi-Infinite Plate Welded to a Rigid Stiffener, Proc. Jap. Civ. Eng., Vol. 314, 149-157.

14. Hasebe N., Okumura M., Nakamura T. (1989), Frictional Punch and Crack in Plane Elasticity, ASCE, J Eng. Mech. , Vol. 115, 1127-1149.

15. Hasebe N., Qian J. (1995), Circular inclined punch problem with Two Corners to Contact with a Half-Plane with a Surface Crack, In: Proc. $2^{\text {nd }}$ Int. Conf. Computational Methods in Contact Mechanics., Computational Mechanics Publications, Southampton, Boston, 159-166.

16. Hasebe N., Qian J. (1997), Circular Rigid Punch with One Smooth and Another Sharp ends an a Half-Plane with Edge Crack, Journal of Applied Mechanics, Vol. 64, 73-79.

17. Hasebe N., Qian J. (1998), Edge Crack Due to Circular Rigid Punch in Incomplete Contact, Mechanics of Materials, Vol. 28, 271-279.

18. Hills D.A., Nowell D. (1994), Mechanics of Fretting Fatigue, Kluwer Academic Publishers, Dordrecht/Boston/London.

19. Hills D.A., Nowell D., Sackfield A. (1993), Mechanics of Elastic Contact, Butterworth, Oxford.

20. Keer L.M., Bryant M.D. (1983), A Pitting Model for Rolling Contact Fatigue, Trans ASME, Journal of Lubrication Technology, Vol. 105, 198-205.

21. Keer L.M., Bryant M.D., Haritos G.K. (1982), Subsurface and Surface Cracking Due to Hertzian Contact, Trans ASME, Journal of Lubrication Technology, Vol. 104, 347-357.

22. Muskhelishvili N.I. (1962), Some basic problems of the Mathematical Theory of Elasticity, Nauka, Moscow, (in Russian).

23. Okumura M., Hasebe N., Nakamura T. (1990), Crack due to Weld Shaped Punch With Friction, ASCE, Journal of Engineering Mechanics, Vol. 116, 2173-2185. 
24. Panasyuk V.V., Datsyshyn O.P., Marchenko H.P. (1995), Contact Problem for a Half-Plane with Cracks under Action of a Rigid Punch on its Boundary, Physicochemical Mechanics of Materials , Vol. 31, 7-16.

25. Panasyuk V.V., Datsyshyn O.P., Marchenko H.P. (2000), Stress State of a Half-Plane with Cracks under Rigid Punch Action, International Journal of Fracture, Vol. 101, 347-363.

26. Qian J., Hasebe N. (1997), Circular Rigid Punch on a Semi-Infinite Plate with an Oblique Edge Crack Subjected to Concentrated Forces on Point Dislocations, Proc. Jap. Civ. Eng., Vol. 570, 149-159.

27. Savruk M.P. (1981), Two-Dimensional Problems of elasticity for Bodies with Cracks, Naukova Dumka, Kiev, (in Russian)

28. Savruk M.P., Madenci E., Shkarayev S. (1999), Singular Integral Equations of the Second Kind with Generalized Cauchy-Type Kernels and Variable Coefficients, International Journal of Numerical Methods in Engineering, Vol. 45, 1457-1470.
29. Savruk M.P., Tomczyk A. (2010), Pressure with friction of an Absolutely Rigid Punch on an Elastic Half-Space with Cracks, Physicochemical Mechanics of Materials, Vol. 46, No 3, 5-15.

30. Tomczyk A. (2011), The Effect of Crack on Contact Pressure Distribution in a Plane Problem Accounting for Friction, Acta Mechanica et Automatica, Vol. 5, № 1, 106-113.

The investigations described in this paper are a part of the research project No S/WM/1/13 realized at Białystok University of Technology. 